Original Research

\title{
Hydrogel contained valproic acid accelerates bone-defect repair via activating Notch signaling pathway in ovariectomized rats
}

\author{
Zhou-Shan $\mathrm{Tao}^{1} \cdot \mathrm{Tian}^{\mathrm{Lin}} \mathrm{Li}^{1} \cdot$ Hong-Guang $\mathrm{Xu}^{1} \cdot$ Min Yang ${ }^{1}$ \\ Received: 2 August 2021 / Accepted: 16 November 2021 / Published online: 23 December 2021 \\ (c) The Author(s) 2021
}

\begin{abstract}
The purpose was to observe whether valproic acid (VPA) has a positive effect on bone-defect repair via activating the Notch signaling pathway in an OVX rat model. The MC3T3-E1 cells were cocultured with VPA and induced to osteogenesis, and the osteogenic activity was observed by alkaline phosphatase (ALP) staining, Alizarin Red (RES) staining and Western blotting (WB). Then the hydrogel-containing VPA was implanted into the femoral epiphysis bone-defect model of ovariectomized (OVX) rats for 12 weeks. Micro-CT, biomechanical testing, histology, immunofluorescence, RT-qPCR, and WB analysis were used to observe the therapeutic effect and explore the possible mechanism. ALP and ARS staining and WB results show that the cell mineralization, osteogenic activity, and protein expression of ALP, OPN, RUNX-2, OC, Notch 1, HES1, HEY1, and JAG1 of VPA group is significantly higher than the control group. Micro-CT, biomechanical testing, histology, immunofluorescence, and RT-qPCR evaluation show that group VPA presented the stronger effect on bone strength, bone regeneration, bone mineralization, higher expression of VEGFA, BMP-2, ALP, OPN, RUNX-2, OC, Notch 1, HES1, HEY1, and JAG1 of VPA when compared with OVX group. Our current study demonstrated that local treatment with VPA could stimulate repair of femoral condyle defects, and these effects may be achieved by activating Notch signaling pathway and acceleration of blood vessel and bone formation.
\end{abstract}

Zhou-Shan Tao

tzs19900327@126.com

1 Department of Trauma Orthopedics, The First Affiliated Hospital of Wannan Medical College, Yijishan Hospital, No. 2, Zhe shan Xi Road, Wuhu 241001 Anhui, People's Republic of China 


\section{Graphical Abstract}

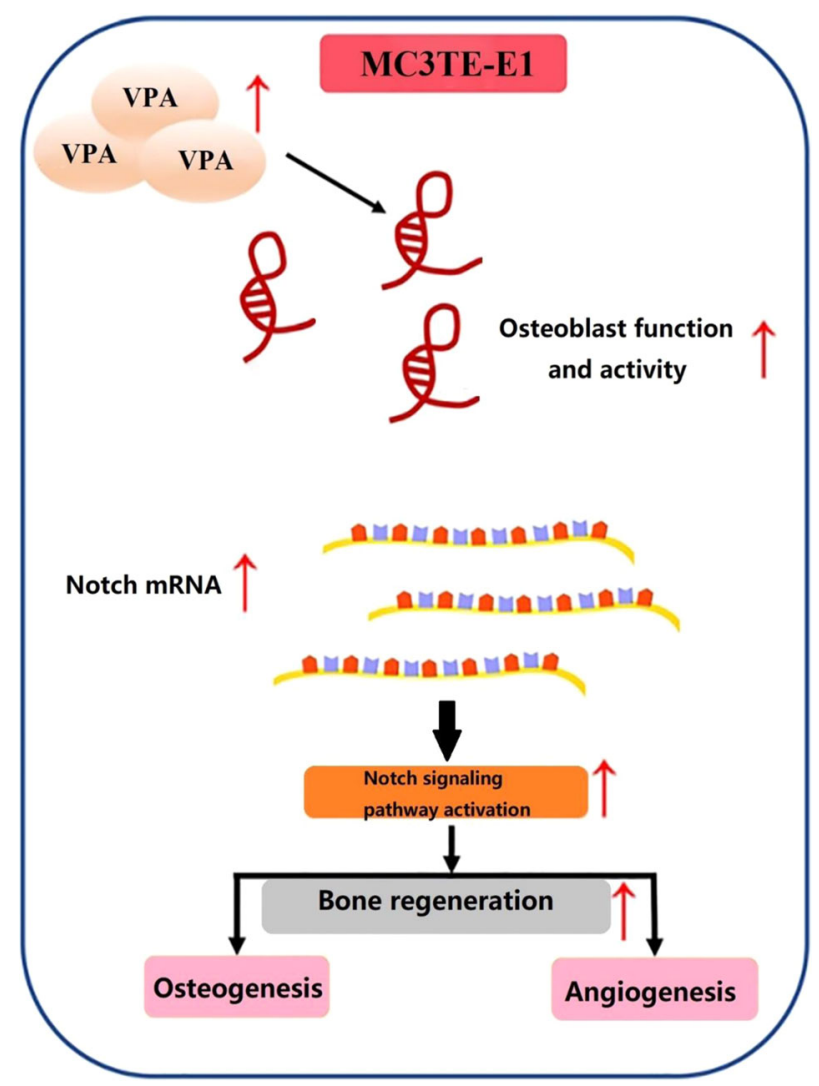

\section{Introduction}

Osteoporosis is a health-concern metabolic bone skeletal disease characterized by increased bone loss and bonestructure deterioration, which will lead to reduced bone mineral density (BMD) and bone strength and increased risk of fragility fractures [1-3]. Most osteoporotic fractures and defects occur in low-energy trauma in elderly people with femoral neck, distal radius and vertebral fractures [4]. In the event of a bone fracture or defects, bone tissue has the unique regeneration ability to replace damaged tissue by constant remodeling with osteoclasts, osteoblasts, osteocytes, and bone-lining cells. When bone defect reaches a certain volume, it is difficult to complete bone-defect healing by its regeneration ability, especially combined with osteoporosis characterized by markedly impaired bone-repair ability [5, 6]. Over the last few decades, bone-substitute materials have received significant attention, and numerous bone biomaterials researches have been reported [7, 8]. While autologous bone graft is still considered to be the "Gold Standard" for bone-defect reconstruction, the complications such as extension of surgical time, increase of surgical sites, and infection chance and aggravation of patient pain may therefore promote people to find and design new alternative drugs and biological materials [9].

Valproic acid (VPA), a branched-chain fatty acid extracted from Valeriana officinalis, which has been widely used in people with epilepsy for more than 20 years owing to its remarkable effect and $80 \%$ oral bioavailability [10]. VPA is an inhibitor of the CYP450 enzyme characterized by minimal effects on hepatic metabolic enzymes that has been used as an antiepileptic drug for many years [11]. Previous studies have reported that VPA functions as a histone deacetylase inhibitor (HDACi), with the specific inhibiting activity by binding to the catalytic center of HDACs, which stimulate apoptosis and inhibit the proliferation of cancer cells [12]. In vitro experimental studies have reported that VPA regulates cell histone acetylation to accelerate osteoblast differentiation and the maturation processes [13, 14]. Interestingly, several cellular and our previous animal experiment have shown VPA's beneficial effects on bone health [15-18].

Although our study has confirmed that VPA plays a positive effect in the process of bone remodeling [19], the local administration of VPA in the treatment of osteoporotic bone defects is lacking and limited. Based on these previous studies and our previous animal experiment, we hypothesized that 
local administration of VPA may have a positive effect on bone-defect regeneration in an OVX rat model. The aim of the present study was to investigate the effect of local treatment with VPA on bone defect in an OVX rat model, and preliminary exploration of possible mechanisms.

\section{Materials and methods}

\subsection{Experimental animal}

The current study employed 40 healthy female SD rats (12 weeks of age, weighing $230 \pm 25 \mathrm{~g}$ ). All animals were housed in groups of five in cages in a temperature- controlled environment $\left(25 \pm 1^{\circ} \mathrm{C}, 55-65 \%\right.$ relative humidity; $12 \mathrm{~h}$ of artificial lighting) in the central laboratory of Yijishan hospital. All experimental rats were kept on pellet feed with standard laboratory diet and tap water, ad libitum. All surgical procedures and drug treatment during the course of the experiment, as well as the sacrifice of the rats at the end of the experiment were approved by the Animal Research Committee.

\subsection{Preparation of VPA hydrogel scaffolds}

The VPA hydrogel was synthesized as described [20]. Briefly, $200 \mu \mathrm{l}$ of poloxamer 407 hydrogel (BASF, Ludwigshafen, Germany) (1/4 volume ratio) was mixed with $50 \mu \mathrm{g}$ of valproic acid and $0.01 \mathrm{M}$ phosphate-buffered saline

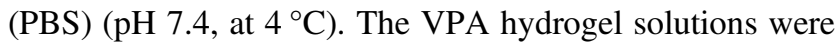
introduced in Teflon molds (1.5-mm diameter $\times 4.0-\mathrm{mm}$ height), and samples were immersed in 0.9 wt.\% of $\mathrm{NaCl}$ solution at $37^{\circ} \mathrm{C}$ for setting.

\subsection{Animal experiments}

In order to establish an osteoporosis model, the rats were subjected to bilateral ovariectomy(OVX, $n=25)$ or sham operation (Sham, $n=15$ ) according to a previously described protocol $[21,22]$ and were kept for 12 weeks. Subsequently, each of five rats from OVX group and Sham group were randomly selected and executed. Bilateral femora were collected and measured by Micro-CT and HE staining to verify the establishment of standard postmenopausal osteoporotic animal models. Then all animals were randomly divided into three groups of 10 rats each: Sham group, OVX group, and VPA-treatment group (VPA group). Once osteoporosis was confirmed, a drilling bone defect with $1.5-\mathrm{mm}$ external diameter of the anteroposterior channel was created by an electric motor with a speed of $1500 \mathrm{rpm}$ in the femoral condyle of the remaining rats according to our previous reports [19, 23]. The rats were classified to VPA group and were implanted and treated with VPA hydrogel. Two intraperitoneal injections of calcein $(20 \mathrm{mg} / \mathrm{kg})$ were injected on the $3 \mathrm{rd}$ and 10 th day before the rats were sacrificed. After 12 weeks of treatment, the rats undergoing bone defect surgery were sacrificed using an overdose of chloral hydrate. Femur samples were harvested. Femurs were fixed at $4{ }^{\circ} \mathrm{C}$ with $4 \%$ paraformaldehyde.

\subsection{Cell culture and alkaline phosphatase (ALP) staining and Alizarin Red (RES) staining}

As an osteoblast precursor cell line, MC3TE-E1 was obtained from the Institute of Biochemistry and Cell Biology, CAS (Shanghai, China). MC3TE-E1 was cultured in 24 -well plates at $1 \times 10^{4}$ cells per well with growth-culture medium. After culturing for $24 \mathrm{~h}$, MC3TE-E1 cells were plated at a density of $1 \times 10^{4}$ cells/ $\mathrm{ml}$ in 24-well plates and cultured in growth medium supplemented with $10^{-8} \mathrm{M}$ dexamethasone (Sigma), $50 \mu \mathrm{g} / \mathrm{ml}$ ascorbic acid (Sigma), and $5 \mathrm{mM} \beta$-glycerol phosphate (Sigma). Then the medium was added with phosphate-buffered saline (PBS) or valproic acid $\left(10^{-6}\right.$ $\mathrm{M})$. The medium was changed every four days during osteogenic differentiation. After induction for 14 and 21 days, osteogenesis was evaluated by staining MC3T3E1 osteoblasts with ALP substrate mixture (ALP staining kit, Sigma) and Alizarin Red reagent (RES, Cyagen Biosciences, Guangzhou, China) as protocol described, respectively.

\subsection{Micro-CT evaluation}

Formation of new bone in defect areas was evaluated by Micro-CT (Bruker Skyscan 1272 system, Kontich, Belgium). The parameter is set to $55 \mathrm{kV}$ and $114 \mathrm{~m} \mathrm{~A}$ with a thickness of $0.048 \mathrm{~mm}$ per slice in medium-resolution mode, 1024 reconstruction matrix, and $200 \mathrm{~ms}$ integration time. These images and parameters of trabecular bone with a distance of $1 \mathrm{~mm}$ proximal from the end of the growth plate in femoral metaphysis were compared between the Sham group and OVX group to confirm the osteoporosis rat model. For evaluation of bone formation in the defect area, a 1.5-mm-diameter area in the center of each bone defect was selected as the volume of interest (VOI). After 3D reconstruction, bone mineral density (BMD), bone mineral content (BMC), bone volume fraction (BV/TV), trabecular number(Tb.N), trabecular thickness (Tb.Th), and trabecular separation(Tb.Sp) were automatically determined for identification of osteoporosis model, while BMD, BV/TV, Tb. $\mathrm{N}, \mathrm{Tb}$. Th, Tb. Sp, and the mean connective density (Conn. D) in VOI regions were used to evaluate new bone formation, using a protocol provided by the manufacturer of the Micro-CT scanner as previously described [24, 25]. 


\subsection{Biomechanical examination}

Compression testing of bone samples was performed immediately after the micro-CT scan. The distal femoral metaphysis of each femur was placed in a $5 \mathrm{~mm}$-wide and $2 \mathrm{~mm}$-deep notch of an aluminum alloy base, which was fixed to the mechanical testing system(Instron 5566; Instron, Norwood, MA, USA). The compression load was applied to the ventral aspect of the condyles at $2 \mathrm{~mm} / \mathrm{min}$ until failure. Ultimate load (N) was calculated from the load-deformation curve.

\subsection{Histomorphometric analysis and immunofluorescence staining}

Part of the femora was decalcified in 10\% EDTA (pH 7.4) for 4 weeks and then embedded in paraffin. Four-micrometer-thick longitudinally oriented along the defect sections were used for staining. The specimens were stained with hematoxylin and eosin (H\&E) according to a standard protocol, viewed under a light microscope and the stained areas were quantified using a BI-2000 medical image analysis system (Chengdu TME Technology Co, Ltd., Chengdu, China). Vascular endothelial growth factor (VEGFA) and recombinant human bone morphogenetic protein-2 (BMP-2) staining were used to quantify the expression of osteogenesis and vascularization factors in the defect area. In brief, fresh bone sections were stained with individual primary antibodies to rats VEGFA (Abcam, ab206887, 1:100) and BMP-2 (Abcam, ab214821, 1:100), overnight at $4{ }^{\circ} \mathrm{C}$. Subsequently, the secondary antibodies conjugated with fluorescence (Jackson Immuno Research, 415$605-166,1: 500 ; 315-605-003,1: 250)$ were used at room temperature for $1 \mathrm{~h}$ while avoiding light and observed under a confocal microscope (FLUOVIEW FV300, Olympus). Calcein double labeling in undecalcified bone slices was observed under a fluorescence microscope (FLUOVIEW FV300, Olympus) to quantify bone mineralization in the defect area.

\subsection{Western blot analysis}

MC3TE-E1 cells 3 days after drug intervention were prepared for Western blotting as previously described. Bone tissue in the defect area was processed with liquid nitrogen. After that, spinOUT columns (GT1200, G-Biosciences, St Louis, USA) were used for the rapid purification of protein. The membrane was incubated with Anti-alkaline phosphatase (ALP, Abcam, ab198554, 1:1000), Anti-RUNX family transcription factor 2 (RUNX 2, Abcam, ab236639, 1:1000), Anti-osteopontin (OPN, Abcam, ab214050, 1:1000), Anti-Osteocalcin (OC, Abcam, ab133612, 1:1000), Anti-VEGFA (Abcam, ab214424, 1:1000), Anti-BMP-2 (Abcam, ab214821, 1:1000), AntiNotch1 (Abcam,ab52627, 1:1000), Anti-HEY1 (Abcam, ab154077, 1:1000), Anti-Jag1 (Abcam, ab109536, 1:1000) and Anti-HES1 (Abcam, ab119776, 1:1000) overnight at $4{ }^{\circ} \mathrm{C}$. Protein expression levels were normalized to Glyceraldehyde 3 phosphate dehydrogenase (GAPDH; Boster, Wuhan, China, 1:2000) protein levels. The next day, the membranes were washed and incubated with the corresponding secondary antibody, diluted at $1: 1000$ for $2 \mathrm{~h}$ at room temperature. The membrane was incubated with ECL-enhanced claim inesence solution and then exposed to X-ray films (Pierce Biotechnology Inc., Rockford, IL).

\subsection{Reverse transcription and real-time polymerase- chain reaction (RT-PCR) analysis}

According to the manufacturer's instructions, total messenger RNA (mRNA) was extracted using the total RNA extraction kit (Takara, Kusatsu, Japan). Complementary DNA (cDNA) was obtained from total RNA using first Strand cDNA Synthesis Kit (Toyobo, Osaka, Japan). Then synthetic cDNAs and specific primers were used for qRT PCR with the TB GreenTM Premix Ex Taq II (Tli RNaseH Plus) kit (Takara, Kusatsu, Japan) on the CFX ConnectTM Real-Time System (Bio-Rad, Singapore). GAPDH was used as an internal control. Sequences of primers for the reference gene (GAPDH) and interested genes are listed in Table 1.

\subsection{Statistical analysis}

All data are shown as mean \pm standard deviation, analyzed using SPSS 19.0 software(IBM SPSS Statistics for Windows, Armonk, NY, USA). One-way analysis of variance(ANOVA) was used for multiple between-group comparisons followed by Tukey's post hoc test. Paired-samples $t$ test was used for comparisons of normal groups and OVX groups. A value of $P \leq 0.05$ was considered to reflect significance.

Table 1 Nucleotide sequences for real-time RT-PCR primers

\begin{tabular}{llll}
\hline Genes & Forward $\left(5^{\prime}-3^{\prime}\right)$ & Reverse $\left(5^{\prime}-3^{\prime}\right)$ & Product length \\
\hline Notch 1 & CGGGGCTAACAAAGATATGC & CACCTTGGCGGTCTCGTA & 68 \\
HES1 & GGAAATGACAGTGAAGCACCT & CAGCACACTTGGGTCTGTG & 78 \\
HEY1 & GGCAGGAGGGAAAGGTTACT & CTCAGATAACGCGCAACTTC & 79 \\
JAG1 & GGCAACACCTTCAACCTCA & GCCTCCACAAGCAACGTATAG & 103 \\
GAPDH & TGCGATGGGTGTGAACCACGAGAA & GAGCCCTTCCACAATGCCAAAGTT & 130 \\
\hline
\end{tabular}


A

ALP
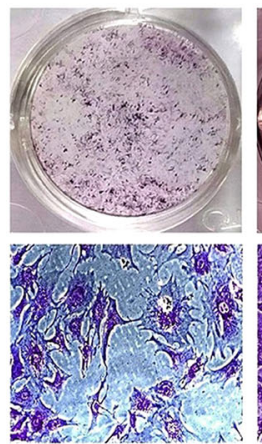

D

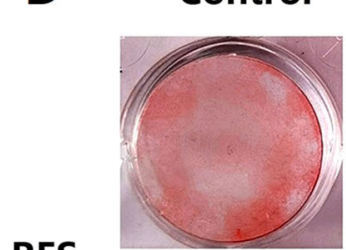

RES

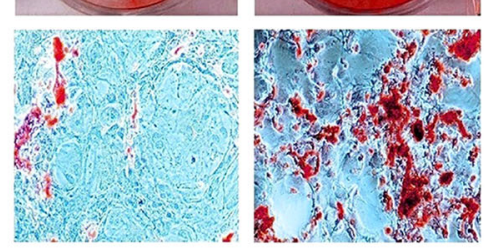

Control

VPA

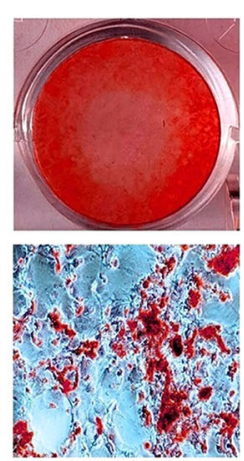

VPA
$\mathbf{G}$

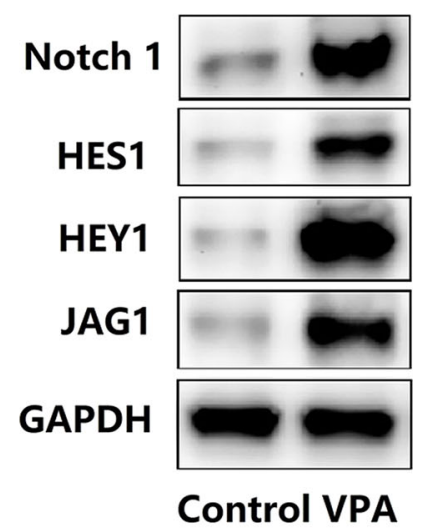

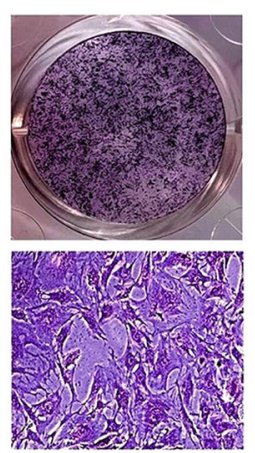

B
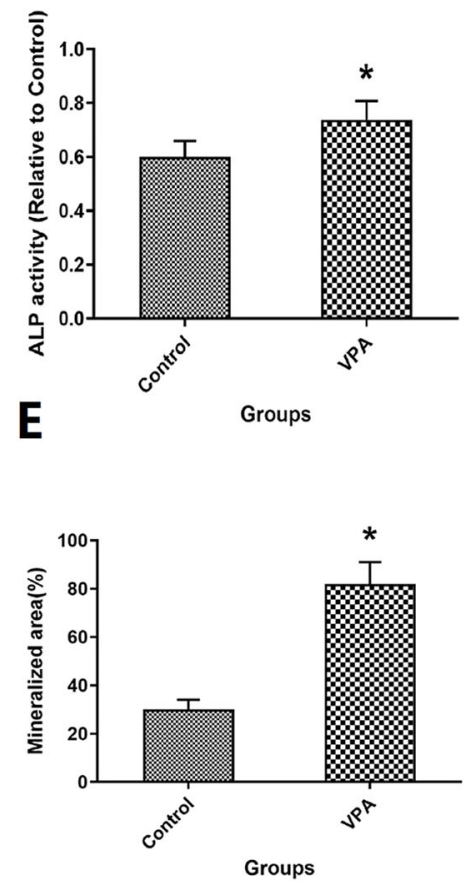

I
H

C
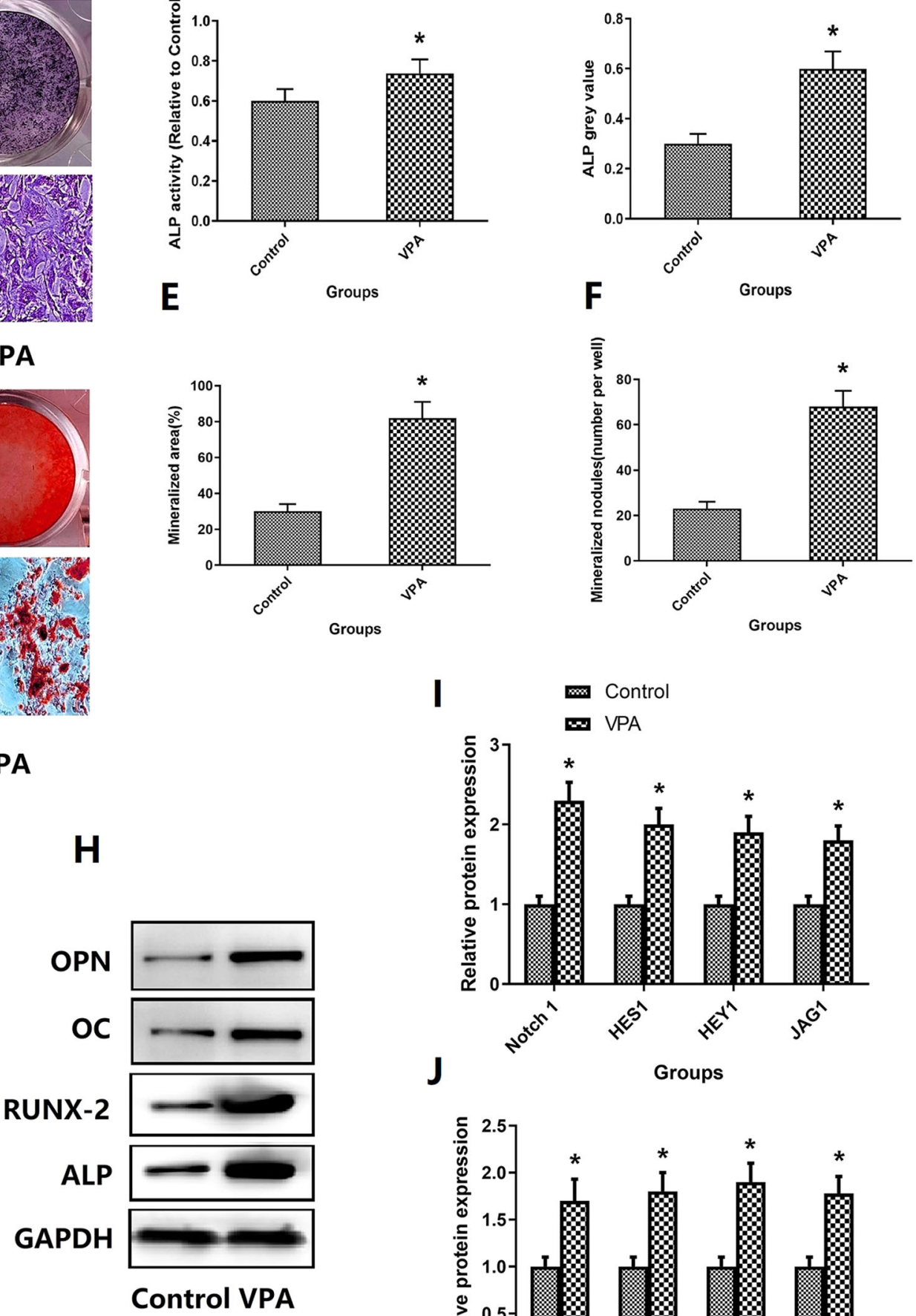

Control

$\infty$ VPA

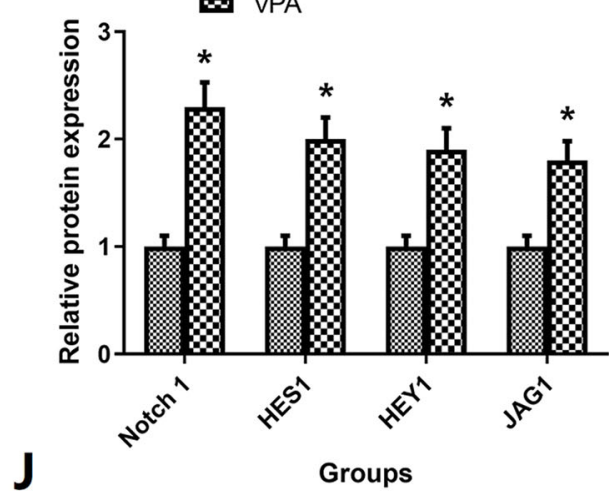

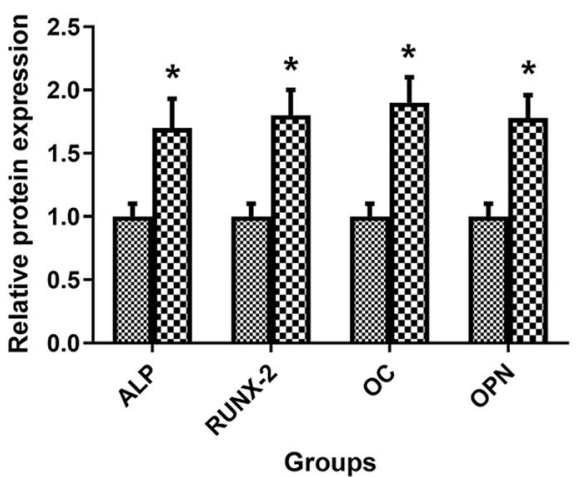

Fig. 1 VPA treatments affect cell function and related protein expression. A, D Representative pictures of ALP staining and Alizarin red staining of osteoblasts after VPA intervention. B, C, E and F The quantification of mineralized nodules, mineralized area, ALP activity,

ALP gray value. $\mathbf{G}, \mathbf{H}, \mathbf{I}, \mathbf{G}$ and $\mathbf{J}$ The relative expression levels of osteoblast-related proteins after VPA intervention and representative pictures of WB detection. *Vs. Control group, $p<0.05$ 


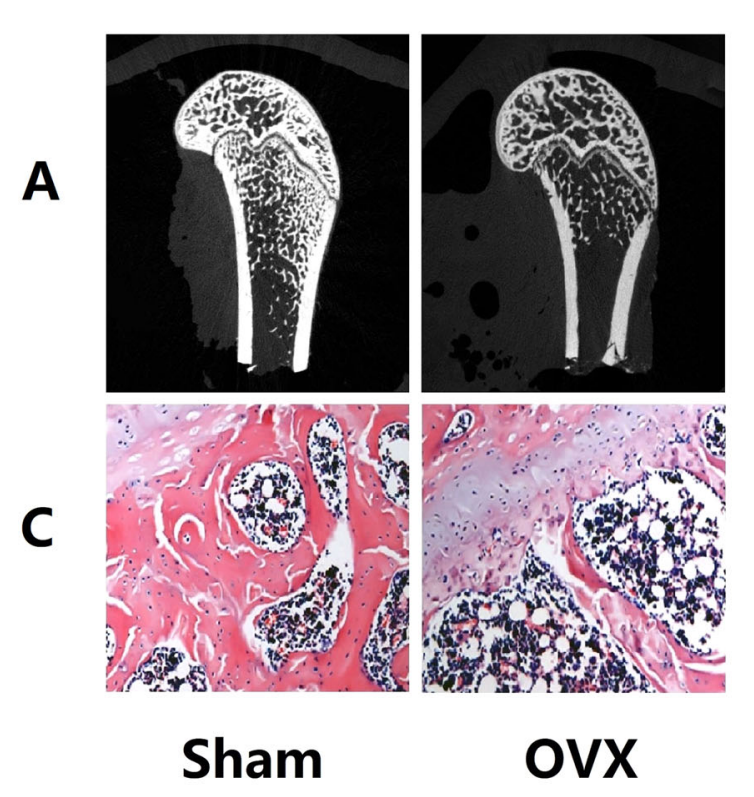

Fig. 2 A 2D micro-CT images of femoral metaphysis in sham and OVX rats, the scale bar represents $1 \mathrm{~mm}$. B The BMD, BMC, BV/TV, Tb.N, Tb.Th, and Tb.Sp of trabecular bone of femoral metaphysis in

\section{Result}

\subsection{Cell function and related protein expression}

In order to determine the effect of VPA on MC3T3-E1 cells function and related protein expression, this study further conducted ALP staining, RES staining and WB analysis. As shown in Fig. 1A, the ALP staining and RES staining with quantification of area in osteogenic differentiation of MC3T3-E1 cells is shown in Fig. 1B. The mineralized nodules (number per well), mineralized area (\%), ALP activity and ALP gray value of VPA group were significantly higher than that of control group $(P<$ 0.05). The osteogenic protein expressions including ALP, OPN, RUNX-2, and OC of VPA group were significantly higher than that of the control group $(P<0.05)$. These results indicate that the treatment with VPA can significantly increase MC3T3-E1 cell function and related protein expression.

\subsection{Osteoporosis animal model validation}

A total of 6 rats died during the experiment, including anesthetic accidents, infection and surgical accidents. No animal death was found in the first operation. The death of rats occurred during or after the second operation, including OVX group $(n=2)$, Sham group $(n=2)$, and VPA group $(n=2)$. After 12 weeks of ovariectomy and sham operation,
B
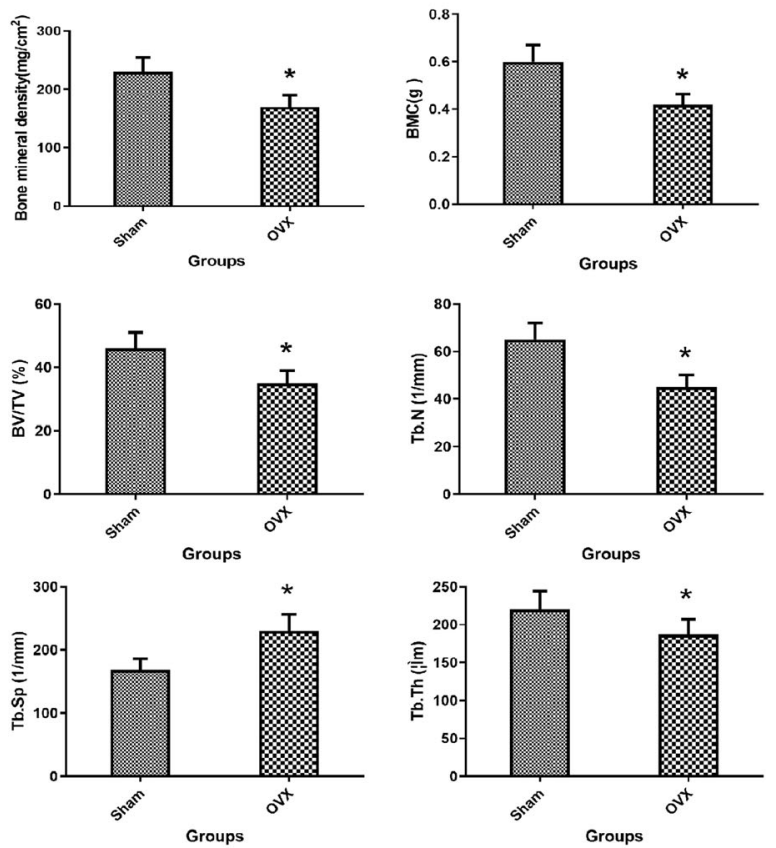

OVX group and sham group. C Representative H\&E staining for the normal and osteoporotic femur(magnification of 20 ). $* P<0.05$ versus Sham group

the femurs of 5 rats were randomly selected and observed by Micro-CT and HE staining microscope as shown in Fig. 2. Imaging and tissue sections clearly show that there is a serious trabecular loss in the metaphysis of the femur in the OVX group; the quantitative results of Micro-CT include BMD, BMC, BV/TV, Tb. Th, Tb. N, and Tb. Sp, which shows that there are significant statistical differences in the above-mentioned indexes between the two groups $(P<0.05)$. These results indicate that the osteoporotic rat model induced by ovariectomized surgery in our experiment achieves the expectation.

\subsection{Micro-CT evaluation}

The 3D reconstruction images and middle part (Fig. 3A-C, $\mathrm{a}-\mathrm{c}$ ) of Micro-CT clearly shows us the bone remodeling of the defect area after 12 weeks of treatment with different intervention methods. As we expected, the defect area of the Sham group was almost filled with bone tissue, while large amounts of bone tissue were found in the VPA group, but it was difficult to find the bone tissue in the OVX group. The quantitative results were expressed as BMD, BV/TV, Tb. Th, Tb. N, Conn. D, and Tb. Sp (Fig. 3). Therapy with VPA showed positive effects on all micro-CT parameters. Compared with group OVX, local treatment with VPS shows better bone microscopic parameters, including the highest BMD, BV/TV, Tb. N, Conn.D, Tb. $\mathrm{Th}$, and a lower $\mathrm{Tb}$. Sp $(P<0.05)$. 


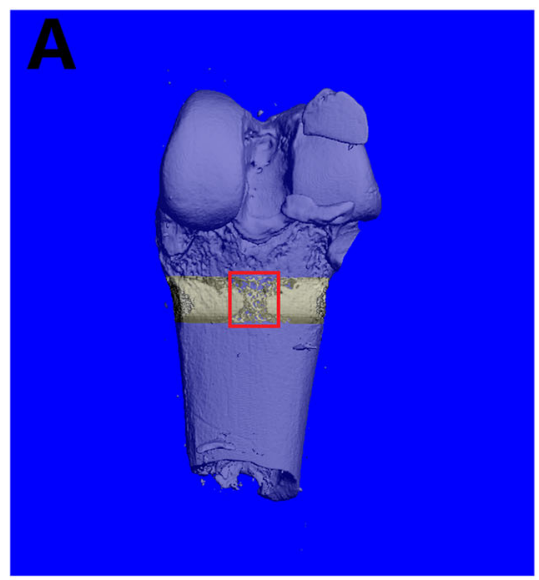

a
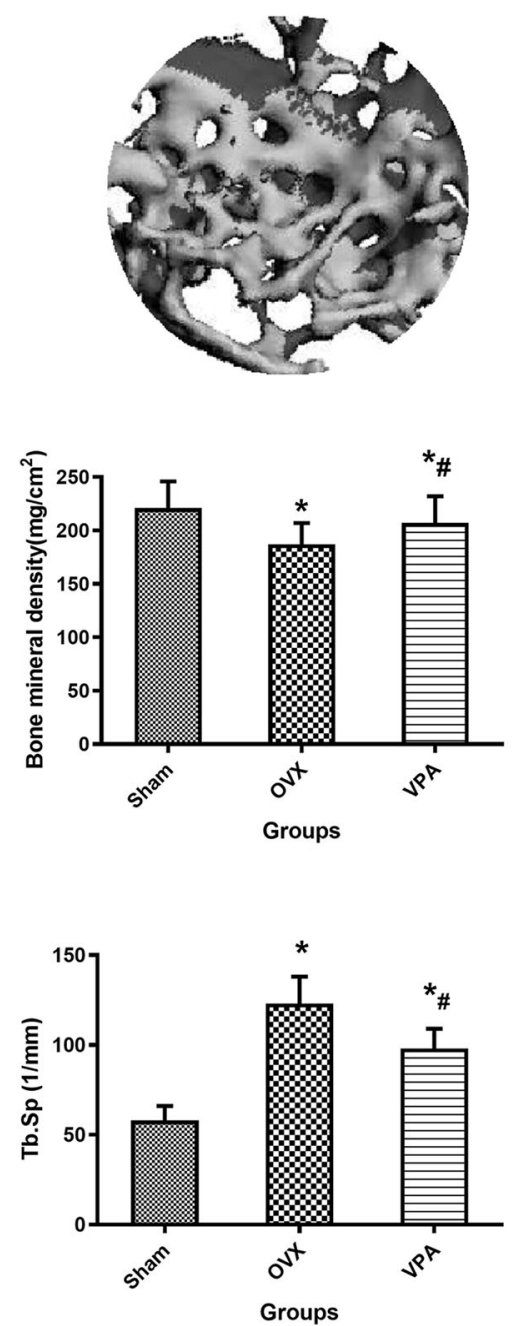

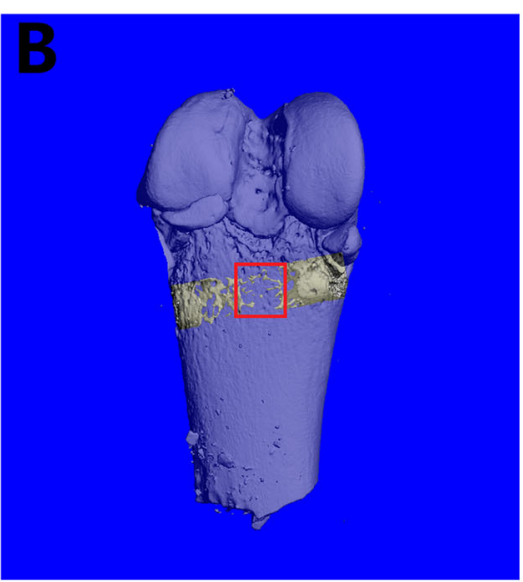

b
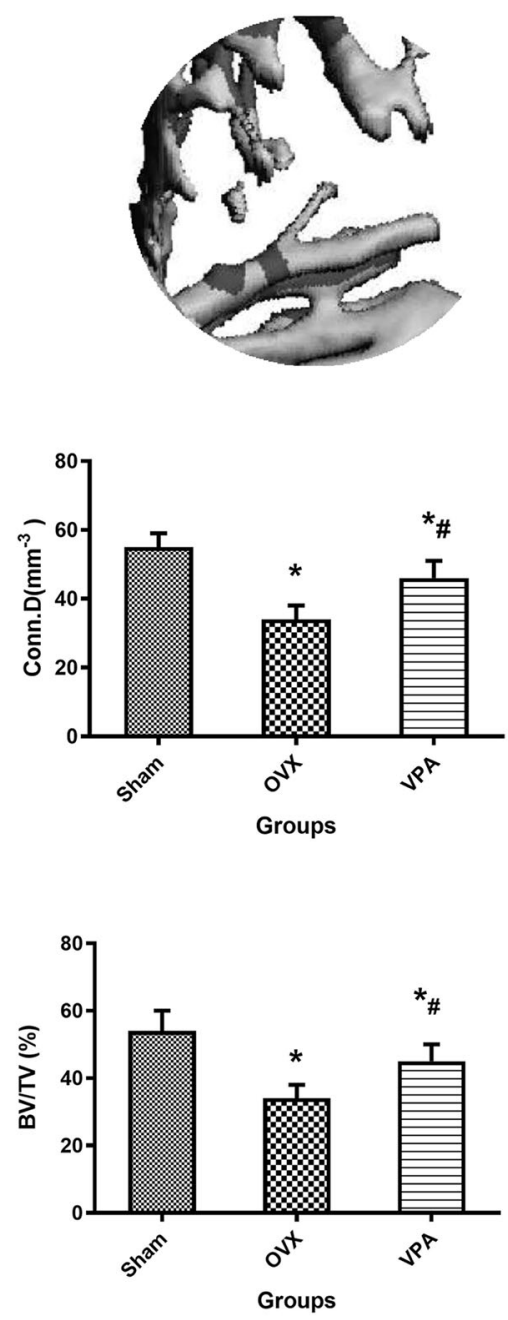

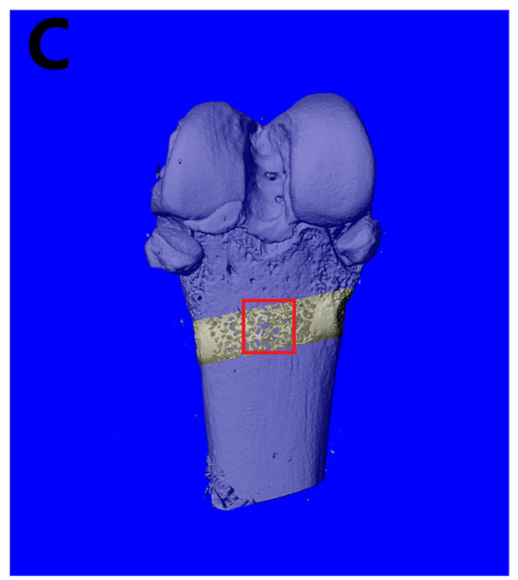

C
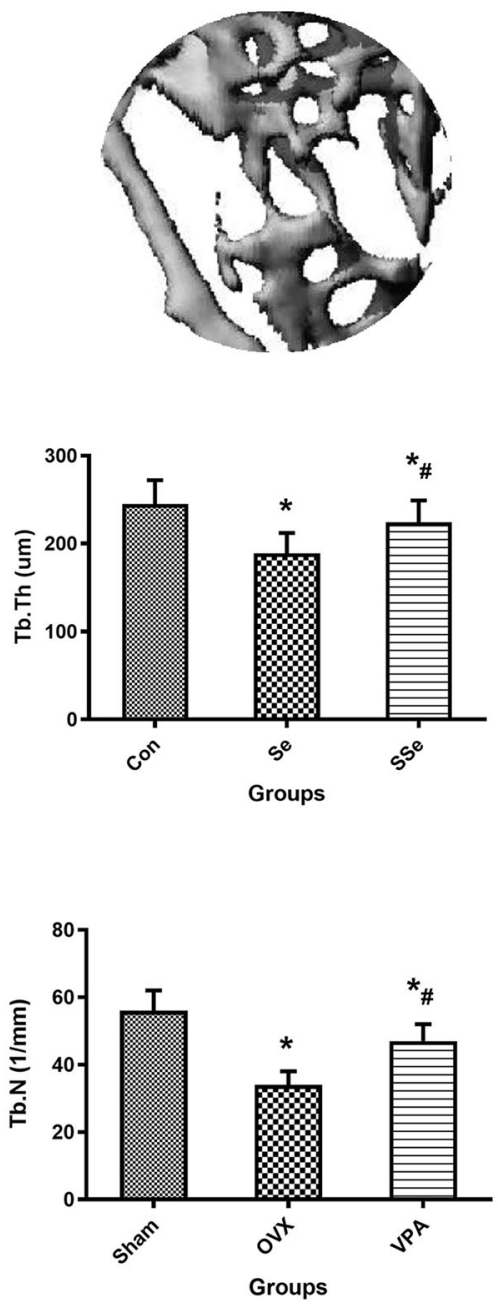

Fig. 3 The representative pictures of Micro-CT images of the distal femur 12 weeks after biomaterials implanted from group of Sham (A, a), $\operatorname{OVX}(\mathbf{B}, \mathbf{b})$, and VPA $(\mathbf{C}, \mathbf{c})$. The scale bar represents $2 \mathrm{~mm}$.
Quantitative results of new trabeculae bone in defect area including BMD, BV/TV, Tb. N, Conn.D, Tb. Th, and Tb.Sp. *Vs. Sham group, $p<0.05,{ }^{\#}$ Vs. OVX, $p<0.05$ 


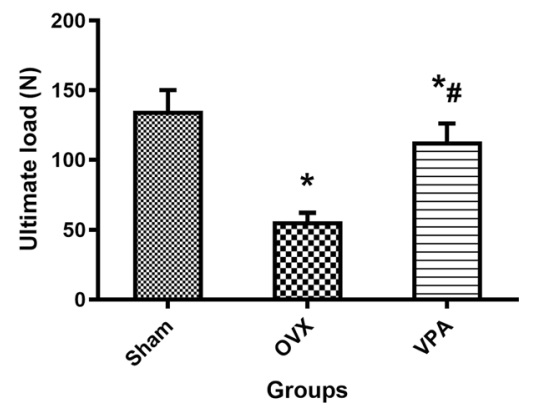

Fig. 4 Biomechanical testing results after VPA treatment. *Vs. Sham group, $p<0.05,{ }^{\#}$ Vs. OVX, $p<0.05$

A

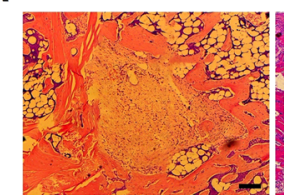

Sham

B

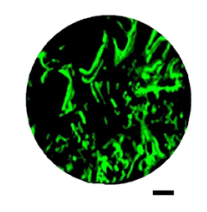

Sham

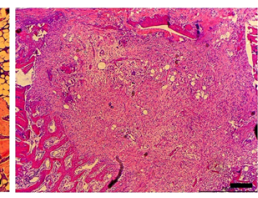

ovx

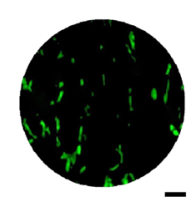

ovx

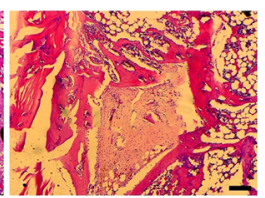

VPA

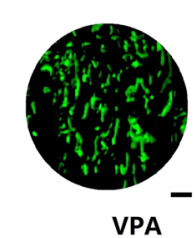

D

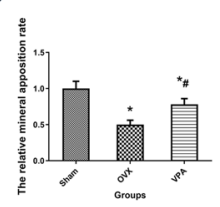

Fig. 5 Bone regeneration of defected area by histological (A, magnification, $\times 10)$ and fluorescent analysis $(\mathbf{B}$, magnification, $\times 200)$. C, D Total fluorescently marked defect area $(\%)$ and relative bone mineralization (green/green marked defect area) after treatment. *Vs. Sham group, $p<0.05$, "Vs. OVX, $p<0.05$

\subsection{Biomechanical testing, histological and fluorescent analysis}

Biomechanical testing, histological, and fluorescent images showing bone repair in defect for different treatments, as shown in Figs. 4 and 5. In 12 weeks, a large amount of bone tissue fills the defect area in the Sham group and VPA group. In the OVX group, only a very small amount of new bone tissue can be observed, and large defect areas still exist. The biomechanical test results show that the ultimate strength of the VPA group is significantly higher than that of the OVX group $(p<0.05)$. In fluorescent analysis, local treatment with VPA showed the larger calcein green-marked defect area $(p<0.05)$, and VPA treatment exhibited the higher values of relative bone mineralization (green/green-marked defect area) $(p<$ $0.05)$, compared to that of the OVX group.

\subsection{Immunofluorescence and RT-PCR, WB analysis of osteogenesis and angiogenesis-regulatory factors}

The osteogenesis and angiogenesis regulator of bone defect measured by immunofluorescence and WB clearly show us the expression of VEGFA and BMP-2 of the defect area after 12 weeks of treatment with different intervention methods (Figs. 6 and 7). As we expected, the defect area of the VPA group was almost filled with immunofluorescence for VEGFA and BMP-2, but it was difficult to find immunofluorescence in the OVX group.

The quantitative results measured by WB were expressed as Notch 1, HES1, HEY1, JAG1, OC, OPN, RUNX-2, ALP, VEGFA and BMP-2. Therapy with VPA showed positive effects on Notch 1, HES1, HEY1, JAG1, OC, OPN, RUNX2, ALP, VEGFA and BMP-2 expression. Compared with group OVX, local treatment with VPA shows the higher protein expression with Notch 1, HES1, HEY1, JAG1, OC, OPN, RUNX-2, ALP, VEGFA, and BMP-2 $(P<0.05)$.

Gene expression of defect area bone tissue after different treatment, as shown in Fig. 7. At 12 weeks, the VPA group showed increased Notch 1, HES1, HEY1, and JAG1 than the OVX group $(p<0.05)$. These results indicate that the Notch pathway of VPA treatment is activated, and the expression of Notch 1, HES1, HEY1, and JAG1 is upregulated.

\section{Discussion}

In this experimental study, a standard osteoporotic animal model was established 12 weeks after bilateral ovariectomy. Bone-forming capacity was evaluated with local administration with VPA in OVX rats for 12 weeks after distal femur-defect creation. The current study provides evidence, by biological activities of MC3T3-E1 osteoblasts, MicroCT, Western blot, real-time PCR, biomechanical testing, histological, and immunofluorescence analyses, confirming the positive effects on bone of local VPA therapy in OVX rats. In addition, the harmful effects on bone regeneration after bone injury were more obvious in OVX rats compared with Sham group rats. After local treatment with VPA, osteogenic ability and bone microstructure parameters of defects were significantly improved in OVX rats. Therefore, our findings indicated that VPA reversed the effects of estrogen deficiency on repair, strengthened the boneregeneration capacity, and enhanced the repair of femoral metaphyseal defects in OVX rats.

In this study, we used standard bilateral OVX rats, the most frequently used hormone-deficient osteoporosis animal 
Fig. 6 After VPA intervention, the relative expression levels of VEGFA and BMP-2 in the bone defect area were detected by immunofluorescence and the representative pictures of $\mathrm{WB}$ detection. A VEGFA and BMP2 expression measured by immunofluorescence; $\mathbf{B}, \mathbf{C}$ immunofluorescence was used to detect the quantitative results of VEGFA and BMP-2 expression in the tissues of the bone-defect area; $\mathbf{D}$ the representative pictures of WB detection; $\mathbf{E}$ the quantitative results of VEGFA and BMP-2 expression in the tissues of the bone-defect area. *Vs. Sham group, $p<0.05,{ }^{\#}$ Vs. OVX, $p<0.05$

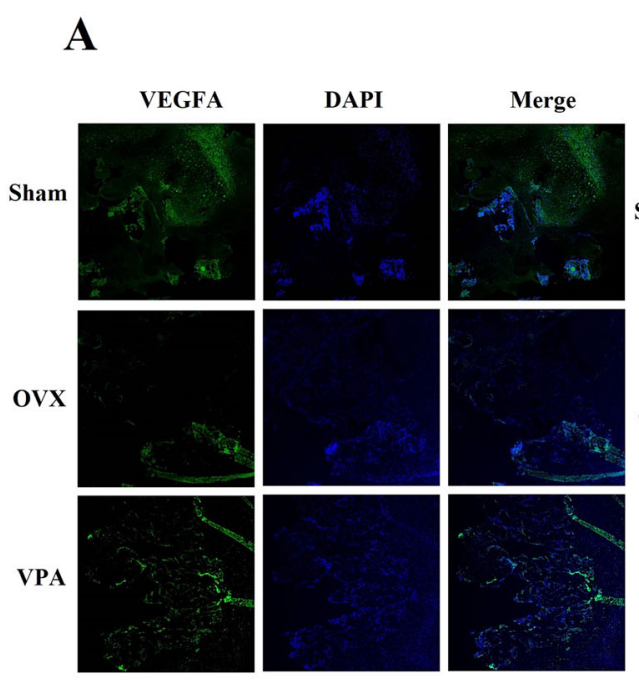

B

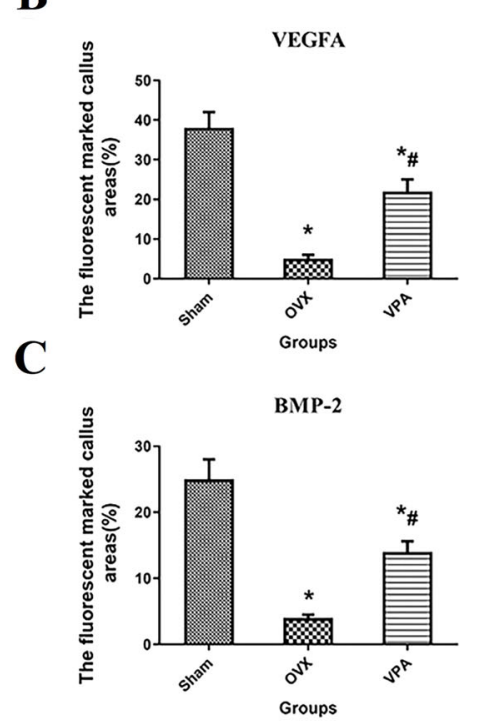

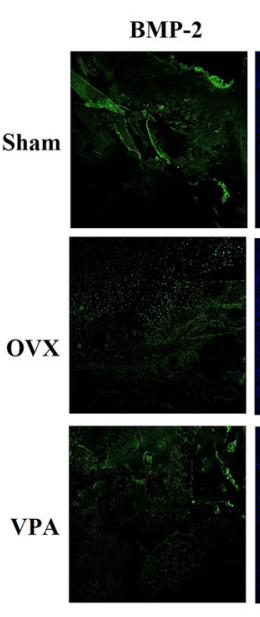
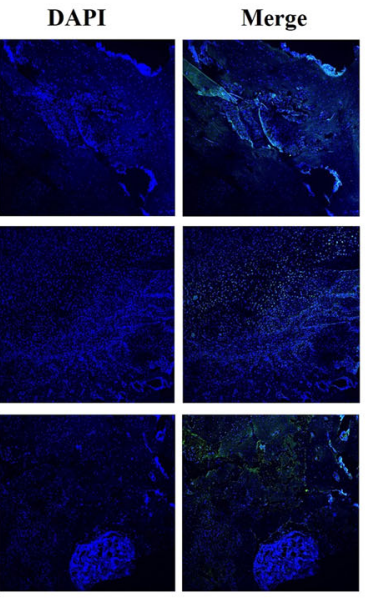

D

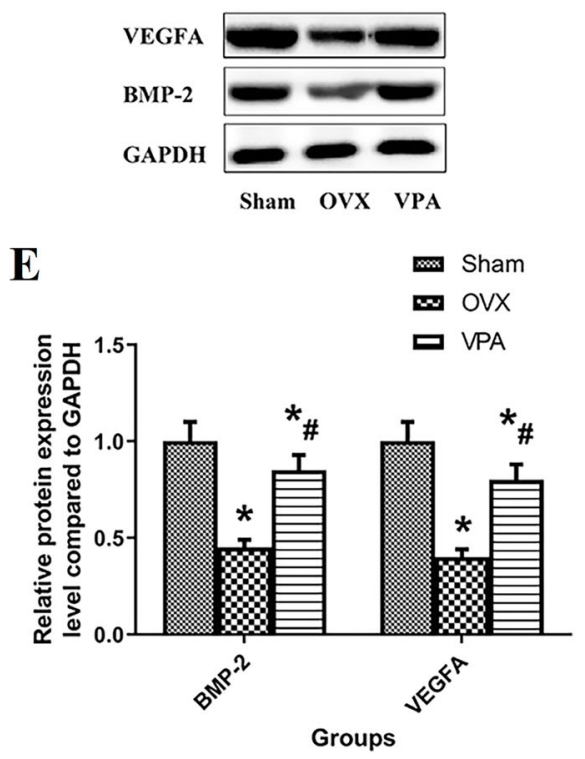

model [26], to mimic osteoporosis observed in menopausal women, and then a hydrogel containing VPA implanted into the femoral condyle defect under the state of osteoporosis to simulate the state of bone defect in the human body. Interestingly, a similar phenomenon that the femoral condyle defect in OVX rats provide a poor bone regeneration with osteoporotic bone was also reported in our previous studies [27, 28]. However, osteoporosis stimulation decreased bone formation and bone repair when compared with the Sham group. These results above confirm that the bone regeneration of bone defects is negatively influenced by osteoporotic bone conditions. Therefore, in order to increase bone-formation potential further, it is necessary to increase bone-regeneration capacity in the state of osteoporosis.

Hydrogel, a kind of three-dimensional, insoluble hydrophilic polymer with good biocompatibility, biodegradability, large water content, and tissue-like flexibility, is used to form drug-delivery vehicles [29]. This material is able to transport nutrients and metabolites from the extracellular matrix for its special structure, which contributes to cell proliferation and differentiation [30], and leads to its wide use in tissue engineering. Currently, a vast array of hydrogels have been investigated, including alginate, fibrin, chitosan, hyaluronic acid, and gelatin. Previous research has confirmed that poloxamer 407 hydrogel is an effective controlled delivery system, and can be competent as a local drug carrier [31]. Therefore, poloxamer 407 hydrogel was used as an effective carrier for VPA in this study. In the study, local treatment with hydrogel containing VPA produced anabolic effects on new bone in femoral condyle defect. Recent studies have shown interesting results for this drug in bone metabolism and bone remodeling, suggesting 


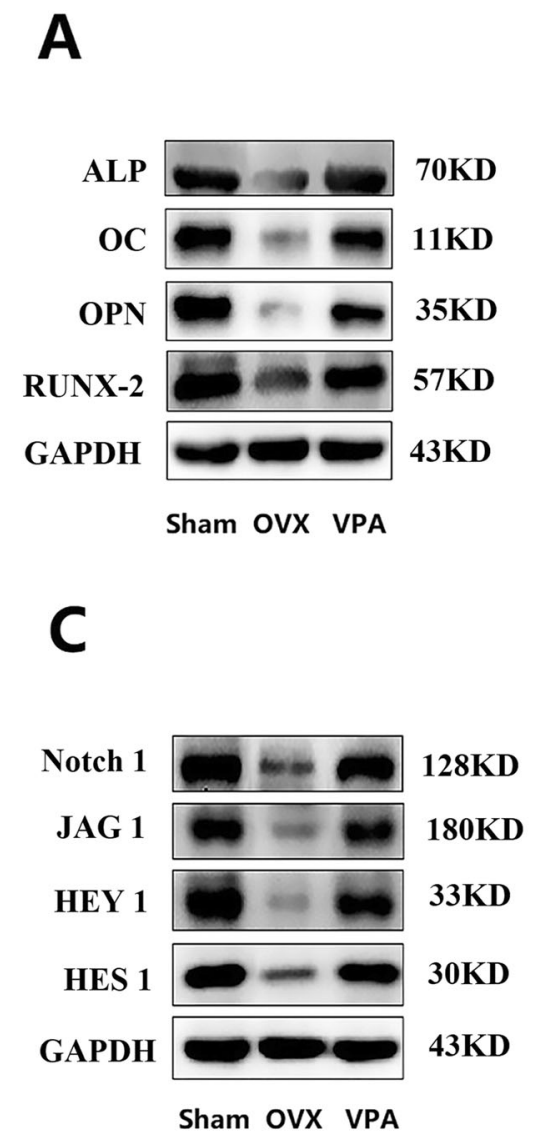

$\mathbf{E}$
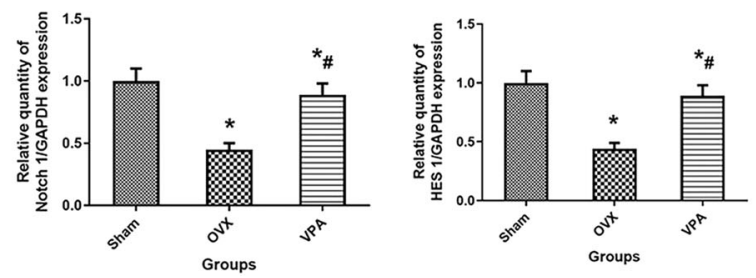

Fig. 7 Protein and gene expression of defect area bone tissue after different treatment. A, B WB detection results and quantitative detection results of osteoblast-related regulatory protein expression. $\mathbf{C}$, D WB detection results and quantitative detection results of specific

that this medication might be relevant for the treatment of osteoporosis, since it has been shown to be effective in preventing bone loss [15-17]. Moreover, recent studies have shown the role of VPA in pathogenesis, tissue regeneration, and can serve as a useful Notch pathway activator and that it is a potential alternative drug for regulation of cell histone acetylation [17]. Indeed, a study has shown that this drug reduces local tissue damage in glucocorticoid-induced osteonecrosis of the femoral head [15]. Previous research has confirmed that intermittent treatment with VPA resulted in significant increases in trabecular thickness and trabecular number while decreasing trabecular separation, which
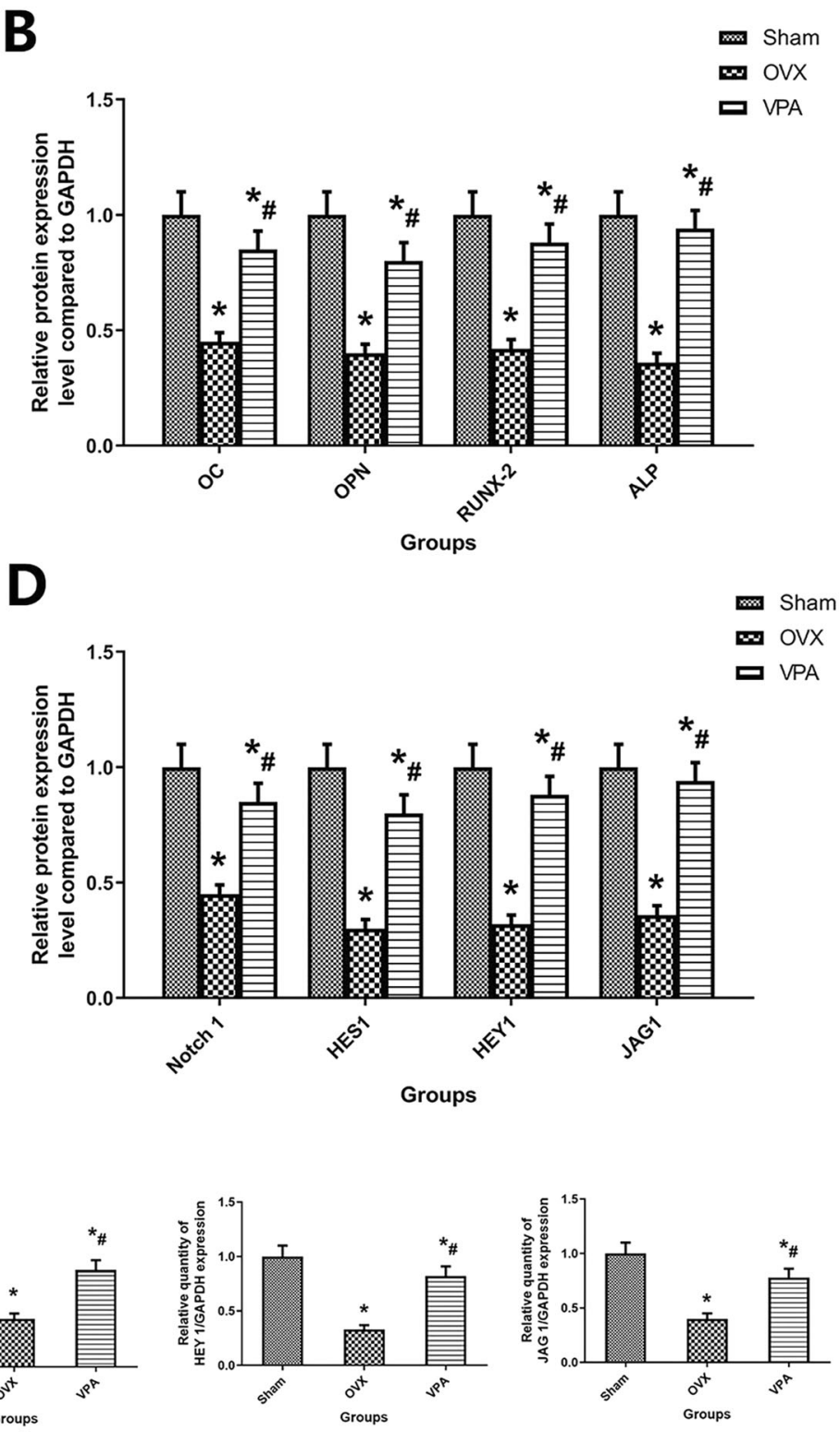

protein expression of Notch pathway. E The quantitative detection results of specific gene expression of Notch pathway. *Vs. Sham group, $p<0.05,{ }^{\#}$ Vs. OVX, $p<0.05$

improved the extrinsic biomechanical properties of bone, prevention of bone fragility, and decreased fracture risk. In vitro cell research has been conducted to evaluate the effect of VPA on biological function of osteoblasts and osteoclasts [16]. Our current results showed that local treatment with VPA increased bone repair and enhanced bone regeneration for 12 weeks.

Why did local treatment with VPA show a positive effect on bone formation in the defect area? Therefore, further investigations are required to confirm these findings and explore possible mechanisms for the observed association. In order to further investigate the potential mechanisms, 
we also performed bone-tissue immunofluorescence, qRTPCR, and western blotting experiments to analyze the mRNA expression of related gene, regulatory factor, and protein contents. Recently, studies indicated that notch signaling also plays a vital role in mineralization of bone tissue via a direct regulation effect on osteoblastic activity [32, 33]. Besides, a large body and animal of emerging evidence has proved that angiogenesis plays a key role in bone repair [34, 35]. Similarly, angiogenesis and osteogenesis were regulated by a variety of growth factors, such as VEGFA and BMP-2 [36, 37]. In this study, the influences of VPA on function of osteogenic biological MC3T3-E1 cells were further detected. As we hypothesized, VPA can significantly promote ALP expression and mineralization in MC3T3-E1 osteoblasts as assessed by ALP staining and Alizarin red staining. The results of WB and RT-PCR show that the dose of VPA used in this study can significantly promote the expression of cellular osteogenic regulatory proteins, such as ALP, RUNX-2, OPN, BMP-2, and OC. In addition, it can activate the Notch pathway and significantly upregulate Notch 1, HES1, HEY1, and JAG1 in MC3T3-E1 osteoblasts and bone tissues. Bone-tissue immunofluorescence and WB clearly showed us the expression of VEGFA and BMP-2 in bone tissue in the defect area, which further confirmed that local blood-vessel formation and bone formation were significantly improved after VPA intervention. Combining the above results, what we can explain is local treatment with VPA can markedly promote osteogenesis and angiogenesis, which causes acceleration of local bone formation and enhanced mineralization ability, and resulting in achieved finally bone regeneration and improved bone strength.

As far as we know, this is the first study of the effect of local administration with VPA on the regeneration of bone defect under osteoporotic conditions. Nevertheless, this study had several deficiencies. The mechanisms underlying the effects of VPA on osteogenic differentiation of MSCs should be elucidated. Delivery systems that allow the sustained release of VPA should be developed for effective bone regeneration in vivo. The optimal dosage of VPA should be determined for bone regeneration by using animal studies.

\section{Conclusion}

In summary, our study suggests that the treatment solution with local administration with VPA is useful to improve the initial bone regeneration of defects by increasing bone formation and angiogenesis in osteoporotic rats. Besides, this benefit effect may be mediated by locals used with VPA via notch signaling pathway.
Acknowledgements This study was supported by a grant from National Natural Science Foundation of China (82002322), Funding of "Peak" Training Program and "Panfeng" Innovation Team Project for Scientific Research of Yijishan Hospital, Wannan Medical College (grant no. GF2019G04, PF2019005, GF2019T02, and PF2019007), and Young and Middle-aged Key Project of Wannan Medical College (WK2020ZF16).

\section{Compliance with ethical standards}

Conflict of interest The authors declare no competing interests.

Publisher's note Springer Nature remains neutral with regard to jurisdictional claims in published maps and institutional affiliations.

Open Access This article is licensed under a Creative Commons Attribution 4.0 International License, which permits use, sharing, adaptation, distribution and reproduction in any medium or format, as long as you give appropriate credit to the original author(s) and the source, provide a link to the Creative Commons license, and indicate if changes were made. The images or other third party material in this article are included in the article's Creative Commons license, unless indicated otherwise in a credit line to the material. If material is not included in the article's Creative Commons license and your intended use is not permitted by statutory regulation or exceeds the permitted use, you will need to obtain permission directly from the copyright holder. To view a copy of this license, visit http://creativecommons. org/licenses/by/4.0/.

\section{References}

1. Zhu Y, Huang Z, Wang Y, Xu W, Chen H, Xu J, et al. The efficacy and safety of denosumab in postmenopausal women with osteoporosis previously treated with bisphosphonates: a review. J Orthop translation. 2020;22:7-13. https://doi.org/10.1016/j.jot. 2019.08.004

2. Zhang S, Huo S, Li H, Tang H, Nie B, Qu X, et al. Flufenamic acid inhibits osteoclast formation and bone resorption and act against estrogen-dependent bone loss in mice. Int Immunopharmacol. 2020;78:106014. https://doi.org/10.1016/j.intimp. 2019.106014

3. Shahrezaee M, Oryan A, Bastami F, Hosseinpour S, Shahrezaee MH, Kamali A. Comparative impact of systemic delivery of atorvastatin, simvastatin, and lovastatin on bone mineral density of the ovariectomized rats. Endocrine. 2018;60:138-50. https:// doi.org/10.1007/s12020-018-1531-6

4. Zhang C, Zhu J, Jia J, Guan Z, Sun T, Zhang W, et al. Onceweekly parathyroid hormone combined with ongoing long-term alendronate treatment promotes osteoporotic fracture healing in ovariectomized rats. J Orthop Res. 2020. https://doi.org/10.1002/ jor. 24953

5. Tao Z, Zhou W, Wu X, Lu H, Ma N, Li Y, et al. Local administration of aspirin improves osseointegration of hydroxyapatite-coated titanium implants in ovariectomized rats through activation of the Notch signaling pathway. $\mathbf{J}$ Biomater Appl. 2020;34:1009-18. https://doi.org/10.1177/ 0885328219889630

6. Zhang W, Shi W, Wu S, Kuss M, Jiang X, Untrauer JB, et al. 3D printed composite scaffolds with dual small molecule delivery for mandibular bone regeneration. Biofabrication. 2020;12:035020. https://doi.org/10.1088/1758-5090/ab906e

7. Vaquette C, Bock N, Tran P. Layered antimicrobial selenium nanoparticle-calcium phosphate coating on 3D printed scaffolds 
enhanced bone formation in critical size defects. ACS Appl Mater interfaces. 2020;12:55638-48. https://doi.org/10.1021/a csami.0c17017

8. Madsen R, Nam D, Schilcher J, Dvorzhinskiy A, Sutherland J, Bostrom F, et al. Mechanical instability induces osteoclast differentiation independent of the presence of a fibrous tissue interface and osteocyte apoptosis in a rat model for aseptic loosening. Acta orthopaedica. 2020;91:115-20. https://doi.org/10.1080/ 17453674.2019.1695351

9. Tao Z, Zhou W, Jiang Y, Wu X, Xu Z, Yang M, et al. Effects of strontium-modified calcium phosphate cement combined with bone morphogenetic protein-2 on osteoporotic bone defects healing in rats. J Biomater Appl. 2018;33:3-10. https://doi.org/10. $1177 / 0885328218765847$

10. Waterhouse E. Intravenous valproate for pediatric status epilepticus. Epilepsy Curr. 2003;3:208-9. https://doi.org/10.1046/j. 1535-7597.2003.03612.x

11. Lee H, Wang S, Salter D, Wang C, Chen S, Fan H. The impact of the use of antiepileptic drugs on the growth of children. BMC pediatrics. 2013;13:211. https://doi.org/10.1186/1471-2431-13-211

12. Insinga A, Monestiroli S, Ronzoni S, Gelmetti V, Marchesi F, Viale A, et al. Inhibitors of histone deacetylases induce tumorselective apoptosis through activation of the death receptor pathway. Nat Med. 2005;11:71-76. https://doi.org/10.1038/nm1160

13. Göttlicher M, Minucci S, Zhu P, Krämer O, Schimpf A, Giavara $S$, et al. Valproic acid defines a novel class of HDAC inhibitors inducing differentiation of transformed cells. EMBO J. 2001;20:6969-78. https://doi.org/10.1093/emboj/20.24.6969

14. Cho H, Park H, Kim Y, Bae Y, Suh K, Jung J. Induction of osteogenic differentiation of human mesenchymal stem cells by histone deacetylase inhibitors. J Cell Biochem. 2005;96:533-42. https://doi.org/10.1002/jcb.20544

15. Zhou D, Chen Y, Yin J, Tao S, Guo S, Wei Z, et al. Valproic acid prevents glucocorticoid-induced osteonecrosis of the femoral head of rats. Int J Mol Med. 2018;41:3433-47. https://doi.org/10.3892/ ijmm.2018.3534

16. Rocha S, Ferraz R, Prudêncio C, Fernandes M, Costa-Rodrigues J. Differential effects of antiepileptic drugs on human bone cells. J Cell Physiol. 2019;234:19691-701. https://doi.org/10.1002/jcp.28569

17. Ji Y, Ke Y, Gao S. Intermittent activation of notch signaling promotes bone formation. Am J Transl Res. 2017;9:2933-44.

18. Tao ZS, Zhou WS, Xu HG, Yang M. Intermittent administration sodium valproate has a protective effect on bone health in ovariectomized rats. Eur J Pharm. 2021;906:174268. https://doi.org/ 10.1016/j.ejphar.2021.174268

19. Tao Z, Zhou W, Jiang Y, Wu X, Xu Z, Yang M, et al. Effects of strontium-modified calcium phosphate cement combined with bone morphogenetic protein-2 on osteoporotic bone defects healing in rats. J Biomater Appl. 2018;33:3-10. https://doi.org/10. 1177/0885328218765847

20. Zhang W, Sun C, Zhu J, Zhang W, Leng H, Song C. 3D printed porous titanium cages filled with simvastatin hydrogel promotes bone ingrowth and spinal fusion in rhesus macaques. Biomater Sci. 2020;8:4147-56. https://doi.org/10.1039/d0bm00361a

21. Yuan Z, Min J, Zhao Y, Cheng Q, Wang K, Lin S, et al. Quercetin rescued TNF-alpha-induced impairments in bone marrow-derived mesenchymal stem cell osteogenesis and improved osteoporosis in rats. Am J Transl Res. 2018;10:4313-21.

22. Tao ZS, Li TL, Wu XJ, Yang M. Local administration with tauroursodeoxycholic acid could improve osseointegration of hydroxyapatite-coated titanium implants in ovariectomized rats. J Biomater Appl. 2021; https://doi.org/10.1177/08853282211027678

23. Li T, Tao Z, Wu X, Yang M, Xu H. Selenium-modified calcium phosphate cement can accelerate bone regeneration of osteoporotic bone defect. J Bone Mineral Metab. 2021; https://doi. org/10.1007/s00774-021-01240-3

24. Li YF, Li XD, Bao CY, Chen QM, Zhang H, Hu J. Promotion of peri-implant bone healing by systemically administered parathyroid hormone (1-34) and zoledronic acid adsorbed onto the implant surface. Osteoporos Int. 2013;24:1063-71. https://doi.org/ 10.1007/s00198-012-2258-5

25. Gabet Y, Kohavi D, Kohler T, Baras M, Müller R, Bab I. Trabecular bone gradient in rat long bone metaphyses: mathematical modeling and application to morphometric measurements and correction of implant positioning. J Bone Miner Res. 2008;23:48-57.

26. Liu H, Yue X, Zhang G. Downregulation of miR-146a inhibits osteoporosis in the jaws of ovariectomized rats by regulating the Wnt/ $\beta$-catenin signaling pathway. Int J Mol Med. 2021;47:1. https://doi.org/10.3892/ijmm.2020.4839

27. Tao ZS, Zhou WS, Wu XJ, Wang L, Yang M, Xie JB, et al. Singledose local administration of parathyroid hormone (1-34, PTH) with beta-tricalcium phosphate/collagen (beta-TCP/COL) enhances bone defect healing in ovariectomized rats. J Bone Min Metab. 2019;37:28-35. https://doi.org/10.1007/s00774-018-0906-3

28. Tao ZS, Lv YX, Cui W, Huang ZL, Tu KK, Zhou Q, et al. Effect of teriparatide on repair of femoral metaphyseal defect in ovariectomized rats. Z Gerontol Geriatr. 2016;49:423-8. https://doi. org/10.1007/s00391-015-0949-1

29. Zhao F, Yao D, Guo R, Deng L, Dong A, Zhang J. Composites of polymer hydrogels and nanoparticulate systems for biomedical and pharmaceutical applications. Nanomaterials. 2015;5:2054-130. https://doi.org/10.3390/nano5042054

30. Syed D, Afaq F, Sarfaraz S, Khan N, Kedlaya R, Setaluri V, et al. Delphinidin inhibits cell proliferation and invasion via modulation of Met receptor phosphorylation. Toxicol Appl Pharmacol. 2008;231:52-60. https://doi.org/10.1016/j.taap.2008.03.023

31. Shafiee A, Kehtari M, Zarei Z, Soleimani M, Varshochian R, Ahmadi A, et al. An in situ hydrogel-forming scaffold loaded by PLGA microspheres containing carbon nanotube as a suitable niche for neural differentiation. Mater Sci Eng C Mater Biol Appl. 2021;120:111739. https://doi.org/10.1016/j.msec.2020.111739

32. AlMuraikhi N, Ali D, Vishnubalaji R, Manikandan M, Atteya M, Siyal A, et al. Notch signaling inhibition by LY411575 attenuates osteoblast differentiation and decreased ectopic bone formation capacity of human skeletal (Mesenchymal) stem cells. Stem Cells Int. 2019;2019:3041262. https://doi.org/10.1155/2019/3041262

33. Cui J, Zhang W, Huang E, Wang J, Liao J, Li R, et al. BMP9induced osteoblastic differentiation requires functional Notch signaling in mesenchymal stem cells. Lab Invest. 2019;99:58-71. https://doi.org/10.1038/s41374-018-0087-7

34. Jiang L, Sheng K, Wang C, Xue D, Pan Z. The effect of MMP-2 Inhibitor 1 on osteogenesis and angiogenesis during bone regeneration. Front Cell Dev Biol. 2020;8:596783. https://doi.org/10. 3389/fcell.2020.596783

35. Chen L, Zhang R, Xie J, Yang J, Fang K, Hong C, et al. STAT3 activation by catalpol promotes osteogenesis-angiogenesis coupling, thus accelerating osteoporotic bone repair. Stem Cell Res Ther. 2021;12:108. https://doi.org/10.1186/s13287-021-02178-Z

36. Sharma S, Xue Y, Xing Z, Yassin M, Sun Y, Lorens J, et al. Adenoviral mediated mono delivery of BMP2 is superior to the combined delivery of BMP2 and VEGFA in bone regeneration in a critical-sized rat calvarial bone defect. Bone Rep. 2019;10:100205. https://doi.org/10.1016/j.bonr.2019.100205

37. Poudel S, Min C, Lee J, Shin Y, Kwon T, Jeon Y, et al. Local supplementation with plant-derived recombinant human FGF2 protein enhances bone formation in critical-sized calvarial defects. J Bone Miner Metab. 2019;37:900-12. https://doi.org/10.1007/ s00774-019-00993-2 